\title{
Observações sobre oviposição, eclosão e tempo de vida de Triatoma matogrossensis Leite \& Barbosa, 1953 (Hemiptera-Reduviidae) em função da alimentação em pombos e coelhos
}

\author{
A study on egg laying, egg hatching and life span of \\ Triatoma matogossensis Leite \& Barbosa, 1953 \\ (Hemiptera-Reduviidae), influenced by blood- \\ meal on both pigeons and rabbits
}

\author{
Ana Maria Marassá, Rosa Maria de 0. Veiga-Barreiros, \\ Roberto Henrique Pinto Moraes, Rute Maria Gonçalves de Andrade, \\ Abel Castillo e Fernando Motta de Azevedo Corrêa
}

\begin{abstract}
Resumo Estudou-se em laboratório a influência de duas diferentes fontes de alimentação sobre oviposição, eclosão dos ovos e tempo de vida de casais de Triatoma matogrossensis. Foram observados 68 casais separados em quatro diferentes grupos, com 20, 12, 20 e 16 casais, respectivamente. Os grupos $A 1$ e $A 2$ foram alimentados em pombos e os grupos $C 1$ e $C 2$ em coelhos. Os casais foram mantidos em estufa a $26^{\circ} \pm 2^{\circ} \mathrm{C}$ e UR de $60-80 \%$, com fotoperíodo de 14 horas. O maior número de ovos postos foi encontrado no grupo A1. Não houve diferença na proporção de ovos eclodidos dos grupos alimentados em coelhos ou pombos. Não foram observadas diferenças de tempo de vida entre machos e fêmeas dos quatro grupos, entretanto o grupo A1 apresentou o maior tempo de sobrevivência e o grupo C2 o menor.
\end{abstract}

Palavras-chaves: Triatoma matogrossensis. Alimentação. Acasalamento. Longevidade.

Abstract A laboratory study was carried out concerning the influence of two kinds of blood-meal on egg laying, egg hatching and life span of Triatoma matogrossensis. 68 couples in 4 different groups with 20, 12, 20 and 16 individuals of each sex per group were formed. Maintained under laboratory conditions groups $A 1$ and $A 2$ were fed on pigeons and groups $C 1$ and $C 2$ were fed on rabbits. In relation to egg laying the best results were found in group $A 1$. No differences on egg hatching were found between the groups fed on rabbits and those fed on pigeons. Concerning the life span, no differences between males and females in the 4 groups were observed but group A1 presented the longest life span and group C2 the shortest.

Key-words: Triatoma matogrossensis. Feeding. Mating. Life span.

\footnotetext{
Laboratório de Artrópodes/Entomologia do Instituto Butantan, Bolsista da Fundação do Desenvolvimento Administrativo (FUNDAP) e Superintendência de Controle de Endemias (SUCEN), São Paulo, SP.

Endereço para correspondência: $\mathrm{Dr}^{\mathrm{a}}$ Ana Maria Marassá. Laboratório de Artrópodes/Entomologia/Instituto Butantan. Av. Vital Brazil 1500, 05503-900 São Paulo, SP, Brasil.

Tel: (011) 813-7222, ramal 2128.

Recebido para publicação em 29/08/97.
} 
É enfocado neste estudo o comportamento de adultos de Triatoma matogrossensis, espécie assinalada por Silveira cols 15 em diversos municípios do Estado de Mato Grosso do Sul, como envolvida na transmissão da doença de Chagas.

Considerações sobre alimentação e cópula são aspectos de relevância em estudos sobre a biologia de triatomíneos. Publicações sobre as espécies Triatoma brasiliensis, Dipetalogaster maximus, Panstrongylus megistus, Rhodnius neivaie Triatoma mazoottii, destacam a influência desses fatores 12389.

Neste estudo avaliou-se o comportamento de adultos de Triatoma matogrossensis, verificando-se, viabilidade de ovos e tempo de sobrevivência em razão de intervalos controlados de alimentação efetuada em coelhos ou pombos, dado que as observações na espécie restringemse ao período ninfal e temperatura13 14 .

\section{MATERIAL E MÉTODOS}

Foram constituídos 68 casais de Triatoma matogrossensis, criados no Laboratório de Artrópodes/Entomologia do Instituto Butantan, desde abril de 1989, a partir de colônia procedente do Instituto de Biociências de Botucatu (UNESP) que vem sendo mantida com repastos efetuados em coelhos e pombos. Os adultos foram isolados após a última muda imaginal, acondicionados e mantidos nos mesmos padrões descritos por Marassá cols 10 .

Formaram-se quatro grupos (A1, A2, C1, C2) constituídos, respectivamente por 20, 12, 20 e 16 casais. Os grupos A1 e A2 foram alimentados em pombos e C1 e C2 em coelhos. Nos grupos $\mathrm{A} 1$ e $\mathrm{C} 1$, o primeiro repasto sangüíneo foi efetuado 3 dias antes do acasalamento e os subseqüentes a intervalos de 14 dias durante toda a observação. Os grupos $\mathrm{A} 2$ e $\mathrm{C} 2$, os casais permaneceram sem alimentação durante as 6 primeiras semanas. Após esse período de jejum, os repastos sangüíneos também foram efetuados a intervalos de 14 dias durante todo o experimento.

Os casais foram observados semanalmente, sendo os ovos coletados e colocados em frascos individualizados até a eclosão das ninfas. $O$ tempo de vida dos adultos foi acompanhado até a morte do último exemplar.

Para cada casal foram observados: o tempo de vida do macho e da fêmea (em semanas); o número de ovos postos por semana antes e após a morte do macho e a proporção de ovos eclodidos antes e depois da morte do macho. Os resultados são descritos segundo as técnicas estatísticas de Análise Descritiva Unidimensional, Análise de Sobrevivência e Análise de Variância com efeitos fixos. A análise estatística foi feita nos dados transformados (log) e as comparações das médias pelo Método de Tukey a nível de significância global de 5\%.

\section{RESULTADOS}

Foram observadas oviposições nos quatro grupos formados. Constatou-se ritmo de postura variável em todos os grupos.

Nos grupos A2 e C2 posteriormente alimentados em coelhos e pombos foram obtidas posturas durante as seis primeiras semanas em que permaneceram sem alimentação. No grupo A2, foram observadas $10(83,3 \%)$ fêmeas com posturas, $8(80 \%)$ delas com posturas férteis, taxa de fertilidade de $71,2 \%$. No grupo C2, foram observadas $11(68,7 \%)$ fêmeas com posturas, sendo férteis em $7(63,6 \%)$ delas com taxa de fertilidade de $55,5 \%$.

Em relação ao número de ovos postos, observou-se no grupo A1 o maior número de ovos postos e no grupo C2 o menor (Tabela 1). Observou-se no grupo $\mathrm{C} 1$ a menor proporção de ovos eclodidos com média abaixo de $30 \%$ (Tabela 2).

Para ovos postos, verificou-se que os 4 grupos apresentaram diferenças $(p=0,0001)$

Tabela 1 - Número médio de ovos postos por Triatoma matogrossensis em função do grupo de tratamento (A1 e A2 alimentados em pombos, C1 e C2 alimentados em coelhos).

\begin{tabular}{|c|c|c|c|c|}
\hline \multirow[t]{2}{*}{ Tratamento } & \multirow[t]{2}{*}{ Total de casais } & \multirow{2}{*}{$\begin{array}{c}\text { Ovos postos } \\
\text { média } \pm \text { D. Padrão }\end{array}$} & \multicolumn{2}{|c|}{ Amplitude } \\
\hline & & & mínimo & máximo \\
\hline Grupo A1 & 20 & $476,20 \pm 281,44$ & 78 & 936 \\
\hline Grupo A2 & 12 & $181,42 \pm 144,29$ & 37 & 453 \\
\hline Grupo C1 & 20 & $104,05 \pm 75,36$ & 7 & 281 \\
\hline Grupo C2 & 16 & $54,31 \pm 55,08$ & 1 & 203 \\
\hline
\end{tabular}


Tabela 2 - Proporção de ovos eclodidos de Triatoma matogrossensis em função do grupo de tratamento.

\begin{tabular}{|c|c|c|c|c|}
\hline \multirow[b]{2}{*}{ Tratamento } & \multicolumn{2}{|c|}{ Proporção de ovos eclodidos } & \multicolumn{2}{|c|}{ Amplitude } \\
\hline & Total de casais & média \pm D. Padrão & mínimo & máximo \\
\hline Grupo A1 & 20 & $0,63 \pm 0,23$ & 0.00 & 0,90 \\
\hline Grupo A2 & 12 & $0,53 \pm 0,34$ & 0,00 & 0,90 \\
\hline Grupo C1 & 20 & $0,27 \pm 0,30$ & 0,00 & 1,00 \\
\hline Grupo C2 & 16 & $0,57 \pm 0,40$ & 0,00 & 1,00 \\
\hline
\end{tabular}

$(F=19,49)$ Grupo A1 > Grupo A2 = Grupo C1 > Grupo C2.

Observou-se que não existe diferença significativa entre as médias dos grupos A2 e C1, no entanto a variabilidade no grupo A2 foi maior.

Para a proporção de ovos eclodidos observouse que os 4 grupos diferem estatisticamente
$(p=0,0041)(F=4,87)$. Ao comparar as médias duas a duas, obteve-se resultado não significativo a nível de $5 \%$ nos grupos A1, A2 e C2, sendo o grupo C1 o único a apresentar comportamento diferente, com uma proporção média bem mais baixa. Nos 4 grupos as médias de ovos eclodidos apresentaram os seguintes valores: grupo $\mathrm{A} 1=0,63 ;$ grupo $\mathrm{A} 2=0,53 ;$ grupo $\mathrm{C} 1=0,27 \mathrm{e}$ grupo $\mathrm{C} 2=0,57$.

Tabela 3 - Medidas descritivas do tempo de vida de Triatoma matogrossenssis (em semanas) segundo o grupo de tratamento.

\begin{tabular}{|c|c|c|c|c|c|}
\hline \multirow[t]{2}{*}{ Tratamento } & \multirow{2}{*}{$\begin{array}{l}\text { Total de exemplares } \\
\text { M/F }\end{array}$} & \multicolumn{2}{|c|}{ Tempo de vida (semanas) } & \multicolumn{2}{|c|}{ Amplitude } \\
\hline & & média & D. Padrão & mínimo & máximo \\
\hline \multirow[t]{2}{*}{ Grupo A1 } & $20 \mathrm{M}$ & 39,20 & 19,55 & 12 & 71 \\
\hline & $20 \mathrm{~F}$ & 39,40 & 20,28 & 10 & 72 \\
\hline \multirow[t]{2}{*}{ Grupo A2 } & $12 \mathrm{M}$ & 30,58 & 17,47 & 8 & 58 \\
\hline & $12 \mathrm{~F}$ & 26,92 & 11,38 & 7 & 43 \\
\hline \multirow[t]{2}{*}{ Grupo C1 } & $20 \mathrm{M}$ & 28,50 & 14,45 & 7 & 52 \\
\hline & $20 \mathrm{~F}$ & 28,60 & 13,37 & 10 & 51 \\
\hline \multirow[t]{2}{*}{ Grupo C2 } & $16 \mathrm{M}$ & 17,50 & 11,09 & 8 & 48 \\
\hline & $16 \mathrm{~F}$ & 15,44 & 8,18 & 7 & 39 \\
\hline
\end{tabular}

A partir do método de estimativas foram construídos os intervalos de confiança para número de ovos postos e proporção de ovos eclodidos nos 4 grupos, apresentados nas Tabelas 4 e 5 .

\begin{tabular}{l} 
Tabela 4 - Intervalos de confiança para o número médio de ovos \\
postos em Triatoma matogrossensis em função do grupo \\
tratamento. \\
\hline Tratamento \\
\hline Grupo 1 \\
Grupos 2 e 3 \\
Grupo 4
\end{tabular}

Em relação ao período de sobrevivência de adultos, o grupo A1 apresentou período de sobrevivência superior ao dos demais grupos e também o maior tempo de vida para machos (71 semanas) e fêmeas (72 semanas; Tabela 3 ).

Tabela 5 - Intervalos de confiança para a proporção média de ovos eclodidos em Triatoma matogrossensis em função do grupo de tratamento.

\begin{tabular}{lc}
\hline Tratamento & Intervalo de confiança \\
\hline Grupos 1,2 e 4 & {$[0,47 ; 0,69]$} \\
Grupo 3 & {$[0,11 ; 0,43]$} \\
\hline
\end{tabular}


Para a análise inferencial do tempo de vida dos insetos foi feita Análise de Sobrevivência utilizando-se o modelo Semiparamétrico de Cox e verificou-se: a) não houve efeito de interação entre sexo e tratamento $(p=0,762)$; b) Não há diferença na sobrevivência de machos e fêmeas $(p=0,429)$; c) o tempo de sobrevivência do Grupo A2 foi semelhante ao do Grupo C1 $(p=0,975)$ e d) o Grupo A1 > Grupo A2 = Grupo C1 > Grupo C2.
O comportamento de todas as variáveis antes da morte do macho foi semelhante ao obtido na avaliação dos casais durante todo o experimento. Deve-se este resultado ao fato das fêmeas terem sobrevivido poucas semanas após a morte dos machos. Conseqüentemente, não foi possível detectar diferenças entre os grupos após a morte dos machos.

\section{DISCUSSÃO}

Diversos autores realizaram observações sobre posturas com diferentes espécies de triatomíneos12 3691216.

Em relação ao Triatoma matogrossensis, observou-se um número médio de ovos/fêmea de 476,2 para os exemplares do grupo A1 e de 104,05 para os do grupo $\mathrm{C} 1$, diferindo estes valores dos resultados obtidos pelos autores acima citados, que observaram menores posturas em exemplares alimentados em ave.

Perondini e cols ${ }^{11}$, Cunha e Brasileiro 5 e Lima cols ${ }^{8}$, em trabalhos realizados com $T$. brasiliensis e $P$. megistus, verificaram a capacidade de fêmeas produzirem ovos sem alimentação. Nos resultados do presente estudo verificou-se uma grande porcentagem de posturas férteis antes do primeiro repasto, o que corrobora as informações desses autores, especialmente as de Lima cols 8 que observaram com $P$. megistus mantidos em jejum durante intervalos de 10 a 54 dias, $5(33,3 \%)$ fêmeas com posturas, $2(40 \%)$ delas com taxa de fertilidade de $90 \%$.

Quanto à taxa de eclosão, diversos autores 12312 verificaram para diferentes espécies uma variação na porcentagem de ovos eclodidos.

Com T. matogrossensis, a proporção média de ovos eclodidos pode ser relacionada apenas com os resultados $(58,7 \%)$ obtidos por Malo cols9 com exemplares de T. mazoottii. Quanto ao numero de ovos postos, exemplares de T. matogrossensis do grupo A2 não diferiram estatisticamente dos do Grupo C1. Quanto ao número de ovos eclodidos, apesar de ter sido observada diferença significativa nos grupos $A 1$ e $C 1$, os demais grupos não apresentaram diferença. De acordo com os resultados obtidos referentes a posturas e eclosões não pode-se afirmar que a dieta influenciou esse comportamento.
Considerando a longevidade média de adultos, Feliciangeli e Rabinovich7 e Rabinovich12 observaram longevidade média de 32,08 e 27,62 semanas para machos e fêmeas de T. maculata e de 26,05 e 16,40 semanas para machos e fêmeas de $T$. infestans. O período de sobrevivência médio dos machos foi maior para as duas espécies e foram observados alguns exemplares com período de sobrevivência atingindo até 72,5 semanas.

Brasileiro ${ }^{1}$ e Souza e cols ${ }^{16}$, com exemplares alimentados em camundongos, observaram uma sobrevivência média de 23,44 semanas para fêmeas de T. brasiliensis e de 40,57 e de 71,85 semanas, respectivamente, para machos e fêmeas de $T$. sordida.

Costa e cols 4 , ao comparar exemplares de D. maximus submetidos a dois regimes de alimentação, obtiveram períodos de sobrevivência de 48,37 e de 18,35 semanas para machos e fêmeas com alimentação em coelhos e de 47,13 e de 18,36 semanas para machos e fêmeas alimentadas em pombos. Os tipos de dieta não influíram nos períodos de sobrevivência dos dois grupos, mas os machos apresentaram maior sobrevivência.

Cabello e cols2, com $R$. neivai, observaram longevidade maior dos exemplares alimentados em coelho $(33,5$ semanas para machos e 36,5 semanas para fêmeas) do que dos alimentados em galinha (23,5 semanas para machos e 24,3 semanas para fêmeas). A dieta teve influência no período de sobrevivência dos dois grupos e a longevidade média das fêmeas foi maior do que a dos machos com qualquer das fontes de alimentação, sendo superior nos exemplares alimentados em coelho.

Com referência à longevidade média, somente os valores obtidos com fêmeas de T. matogrossensis do grupo $\mathrm{C} 1$ ficaram próximos daqueles obtidos por Brasileiro1 com fêmeas de T. brasiliensis permanentemente acasaladas. 
Ao comparar os períodos de sobrevivência nos 4 grupos, que tiveram acesso a diferentes tipos de fonte alimentar verificou-se nos exemplares do grupo A1 o período mais longo e nos do grupo C2 o mais curto. Apesar dos exemplares do grupo A1 terem apresentado o período mais longo não podemos concluir que a dieta influiu sobre o período de sobrevivência pois aqueles pertencentes aos grupos A2 e C1 tiveram comportamento semelhante e intermediário aos dos grupos A1 e C2.

Em relação ao tempo de sobrevivência de machos e fêmeas, nossos resultados divergem daqueles encontrados pelos autores citados, visto que não foram evidenciadas diferenças entre os 4 grupos que constituíram objeto de nossas observações.

\section{REFERÊNCIAS BIBLIOGRÁFICAS}

1. Brasileiro VLF. Fecundidade e fertilidade da fêmea de Triatoma brasiliensis (Hemiptera - Reduviidae). IInfluência da cópula e da longevidade. Revista Brasileira de Biologia 42:1-13,1982.

2. Cabello DR, Lizano E, Valderrama A. Efecto de la frecuencia alimentaria sobre algunos parâmetros poblacionales de Rhodnius neivai. Memórias do Instituto Oswaldo Cruz 83:441-446,1988.

3. Costa JM, Jurberg J, Almeida JR. Estudos bionômicos de Dipetalogaster maximus (Uhler,1894) (Hemiptera-Triatominae). I- Influência da dieta sobre o ritmo de postura, viabilidade dos ovos, curva de fertilidade e mortalidade das fêmeas. Memórias do Instituto Oswaldo Cruz 81:365-380,1986.

4. Costa JM, Jurberg J, Almeida JR. Estudos bionômicos de Dipetalogaster maximus (Uhler,1894) (Hemiptera - Triatominae) II - Influência da dieta sobre o ciclo biológico e resistência ao jejum. Memórias do Instituto Oswaldo Cruz 82:111-118,1987.

5. Cunha CS, Brasileiro VLF. Maturidade sexual e reprodução em fêmeas de Panstrongylus megistus Burmeister,1835 (Hemiptera - Reduviidae) em laboratório. Revista Brasileira de Entomologia 32:471-478,1988.

6. Diotaiiuti L, Dias JCP. Estudo comparativo do ciclo evolutivo de Rhodnius neglectus alimentados em pombos ou camundongos. Revista da Sociedade Brasileira de Medicina Tropical 20:95-100, 1987.

7. Feliciangeli MD, Rabinovich J. Vital statistics of Triatominae (Hemiptera - Reduviidae) under laboratory conditions II - Triatoma maculata. Journal of Medical Entomology 22:43-48,1985.

8. Lima MM, Jurberg P, Almeida JR. Behaviour of Triatomines (Hemiptera-Reduviidae) vectors of Chagas' disease. IV- Fecundity, fertility and longevity of Panstrongylus megistus (Burm, 1835) pairs and virgin females, starved under laboratory conditions.
Memórias do Instituto Oswaldo Cruz 82:501509, 1987.

9. Malo EA, Rovelo AR Lopez LC, Rojas JC. Life cicle and influence of age and feeding on the first mating of Triatoma mazoottii (Hemiptera-Reduviidae). Memórias do Instituto Oswaldo Cruz 88:203-206, 1993.

10. Marassá AM, Veiga-Barreiros RMO, Moraes RHP, Andrade RMG, Zorzenon FJ, Corrêa FMA. Fecundidade de fêmeas de Triatoma vitticeps (Stal, 1859) e viablilidade de ovos em razão do tempo de permanência com os machos. Revista de Ciências Biomédicas 16: 63-70, 1995.

11. Perondini ALP, Costa MJ, Brasileiro VLF. Biologia do Triatoma brasiliensis. II- Observações sobre a autogenia. Revista de Saúde Pública 9:363-370, 1975.

12. Rabinovich JE. Vital statistics of Triatominae (Hemiptera - Reduviidae) under laboratory conditions. I - Triatoma infestans (Klug, 1834). Journal of Medical Entomology 9:351-370,1972.

13. Rangel EF. Observações sobre a influência da temperatura no periodo de incubação dos ovos de Triatominae (Hemiptera - Reduviidae). Anais da Sociedade Entomológica do Brasil 11:255-299,1982.

14. Silva TG. Influência da temperatura na biologia de triatomíneos. VIII - Triatoma matogrossensis Leite \& Barbosa, 1953 (Hemiptera - Reduviidae). Anais da Sociedade Entomológica do Brasil 18:91-94, 1989.

15. Silveira AC, Feitosa VR, Borges R. Distribuição triatomíneos capturados no ambiente domiciliar, no período de 1975/83. Brasil. Revista Brasileira de Malariologia e Doenças Tropicais 6:15-312, 1984.

16. Souza JMP, Rodrigues VLCC, Silva EOR. Triatoma sordida - Considerações sobre o tempo de vida das formas adultas e sobre a oviposição das fêmeas. Revista de Saúde Pública 2:291-296,1978. 